Bhaskaran, K. \& Gorrill, R. H. (1957). J. gen. Microbiol. 16, 721-729

\title{
A Study of Antigenic Variation in Vibrio cholerae
}

\author{
By K. BHASKARAN* aND R. H. GORRILL $\dagger$ \\ The Wright-Fleming Institute of Microbiology, St Mary's Hospital \\ Medical School, London, W. 2
}

\begin{abstract}
SUMMARY: The variations in Vibrio cholerae studied included changes from Ogawa to Inaba type, smooth $\rightarrow$ rough, and motile $\rightarrow$ non-motile. With a sloppy-agar technique it was possible to estimate the rate of formation of these variants (i.e. probable mutation rate 1 in $10^{5}$ and 1 in $10^{4}$, respectively). It was possible to show that the action of antiserum in promoting the change from Ogawa to Inaba was selective rather than mutagenic. With the other two variations studied no selective process had to be considered owing to their high spontaneous rate of appearance. It may be said that all three variations studied were due to spontaneous mutants in the parent cultures giving rise to these forms.
\end{abstract}

The studies of Gardner \& Venkatraman (1935) showed that vibrio species could be separated on the basis of their somatic ' $O$ ' antigens, while the flagellar ' $H$ ' antigens were widely shared. Since then the differentiation of Vibrio cholerae, the pathogen of cholera, from similar cholera-like vibrios, rests on its agglutinability with specific ' $\mathrm{O}$ ' antisera. $V$. cholerae constituted $\mathbf{O}$ group $\mathbf{I}$, in this scheme of classification, along with certain haemolytic 'El Tor' vibrios. The definition of $V$. cholerae as a non-haemolytic vibrio belonging to $\mathrm{O}$ group I (Gardner \& Venkatraman) is now generally accepted, and $\mathrm{O}$ antisera prepared with heat-killed suspensions are almost universally used for the identification of $V$. cholerae, in the absence of any reliable biochemical critera. Gardner \& Venkatraman (1935) also confirmed the observations of early Japanese workers (Kabeshima, 1918; Nobechi, 1923) when they defined subtypes Inaba, Ogawa and Hikojima in $V$. cholerae which, while sharing group-specific $\mathrm{O}$ antigens, differed in minor type-specific $\mathrm{O}$ antigens. Types Inaba and Ogawa were the 'end' types, usually designated by the antigenic symbols $\mathrm{AC}$ and $\mathrm{AB}$ respectively, while Hikojima formed the intermediate type $\mathrm{ABC}$. In view of the poor content of antigen $\mathrm{C}$ in Hikojima strains, as well as its presence in Ogawa type cultures when grown at $20^{\circ}$, Kauffmann (1950) suggested that Ogawa-Hikojima types constituted one form of $V$. cholerae, as distinct from the Inaba form.

Cholera outbreaks in India are usually confined to a single type of Vibrio cholerae in localized areas, though the type may vary from place to place and epidemic to epidemic. Hikojima-type strains seem to be of rare occurrence while Inaba and Ogawa types have contributed to most epidemic outbreaks. The shift between these two types, over several years, in the cholera endemic

* Present address: Central Drug Research Institute, Lucknow, India.

$\dagger$ Present address: Department of Bacteriology, Guy's Hospital Medical School, London, S.E. 1. 
areas of Madras State was summarized by Venkatraman (1947), who observed that a change in antigenic type followed years of comparatively low incidence of cholera.

Changes in antigenic type of Vibrio cholerae under laboratory conditions were observed by Kabeshima (1918) and a detailed study of this phenomenon was made by Shrivastava \& White (1947). They observed that Inaba-type organisms could be consistently isolated from Ogawa-type cultures when the latter were grown in broth containing type-specific Ogawa $\mathrm{O}$ antiserum. A similar expedient of growing Inaba-type cultures in broth containing Inaba $\mathrm{O}$ antiserum yielded only rough forms and not the Ogawa type, except in the case of certain imperfect Inaba strains which appeared to belong in reality to the Hikojima category. This evidence of a one-way antigenic change in V. cholerae probably led Shrivastava \& White (1947) to believe that they resulted from loss variations, presumably through some fault in germinal distribution on cellular division. The present investigation was designed to study the change of Ogawa to Inaba type and certain other antigenic changes, with the aid of a rapid technique which permitted the typing of a large number of colonies at a time without resorting to laborious agglutination tests.

\section{METHODS}

Media. For the cultivation of Vibrio cholerae, the PLYP medium described by Gorrill \& Gray (1956) was used. This contained peptone (Evans), $10 \mathrm{~g}$; Yeastrel (Brewers Food Supply Co. Ltd., Edinburgh, 3), 3 g. ; Lab-Lemco, 5 g.; Pronutrin (Herts Pharmaceuticals Ltd., Welwyn Garden City), 10 g.; and sodium chloride (AR), $5 \mathrm{~g}$.; in $1 \mathrm{l}$. distilled water. The mixture was adjusted to $\mathrm{pH} 8.0$ and autoclaved at $10 \mathrm{lb}$. $/ \mathrm{sq}$.in. for $10 \mathrm{~min}$. Difco agar to $2 \%(\mathrm{w} / \mathrm{v})$ was added to make solid medium when required.

Strains of Vibrio cholerae used. Inaba-type cultures: 243, King Institute of Preventive Medicine, Madras; 8024, National Collection of Type Cultures, Colindale Avenue, London, N.W. 9; 53/54 and 55/54, All India Institute of Hygiene and Public Health, Calcutta.

Ogawa-type cultures: 502, Central Drug Research Institute, Lucknow; M. 64, M. 70, M. 76, All India Institute of Hygiene and Public Health, Calcutta; 8022, National Collection of Type Cultures, Colindale Avenue, London, N.W. 9.

Antisera. High-titre agglutinating $\mathrm{O}$ antisera were prepared by immunizing adult male rabbits with heat-killed cultures of Vibrio cholerae, as carried out by Gardner \& Venkatraman (1935). Monospecific Inaba and Ogawa $\mathbf{O}$ antisera were obtained by absorbing the corresponding sera with heat-killed cultures of the heterologous type. The agglutinin titres of the antisera varied from $1 / 2500$ to $1 / 5000$. The rabbits were re-immunized on five or six successive occasions at 2 - to 3 -monthly intervals with the respective antigens. The $\mathrm{O}$ agglutinin titre was maintained by this means and in a few animals the titre went up to $1 / 10,000$ after repeated immunization.

Procedure for typing colonies of Vibrio cholerae. The antiserum in sloppy-agar technique used by Stocker (1949) to differentiate antigenic phases in Salmo- 
nella typhimurium proved very satisfactory for the differentiation of antigenic types in V. cholerae and will be referred to as 'Stocker's sloppy-agar technique'. The test, performed as below, gave consistent results:

(i) PLYP medium containing $2 \%$ Difco agar, and sloppy PLYP medium containing $0.5 \%$ Difco agar were prepared. The sloppy medium was dispensed in $10 \mathrm{ml}$. amounts in $1 \mathrm{oz}$. screw-capped bottles, while the solid medium was poured into $9 \mathrm{~cm}$. diam. Petri dishes, using about $15 \mathrm{ml}$. medium/dish.

(ii) The culture to be tested was seeded on the agar plate at such a dilution as to give about 200 to 400 colonies/dish.

(iii) When fully developed colonies appeared on the medium after 18-24 hr. of incubation at $37^{\circ}$ the test was performed. To $10 \mathrm{ml}$. of sloppy agar, melted in the steamer and cooled to $50^{\circ}$, was added $0.1 \mathrm{ml}$. of type-specific antiserum (either Inaba or Ogawa) and after mixing well, gently layered on the surface of the culture in the dish. After allowing $10 \mathrm{~min}$. for the agar to set, the plates were transferred to an incubator $\left(37^{\circ}\right)$ and results read after $15 \mathrm{~min}$.

Three different colony appearances were encountered:

(a) 'haloed' colonies: these colonies appeared dense and opaque with a well-pronounced halo round each colony as the organisms diffused out. This appearance was associated with types not agglutinated by the serum used.

(b) 'non-haloed' colonies: these colonies appeared dense and opaque but without a halo. The edge of the colony was clear-cut. This appearance was found with types agglutinated by the serum used.

(c) 'compact' colonies: these colonies differed from the non-haloed type in being thin and translucent. The dense opalescence did not develop on the addition of sloppy agar, nor was there a halo around the colony. This appearance was found in non-motile mutants and in salt-unstable rough mutants. Colonial differentiation between these two was not possible by this method.

The reliability of the above appearances and their correlation with the associated characters was checked by model experiments with type mixtures. The appearance of rough and non-motile mutants in this test was not influenced by the antiserum in the sloppy layer. They showed the same characteristics in antiserum-free sloppy agar which served to differentiate them from smooth motile organisms, and was in fact used to isolate such mutants. When using antiserum in the sloppy layer to differentiate between the types, it was essential to have an adequate concentration of the antiserum. With titres of $1 / 2500$ or more, $0 \cdot 1 \mathrm{ml}$. of undiluted antiserum for $10 \mathrm{ml}$. of sloppy agar was sufficient. Antisera with lower titres were not used in this test.

\section{RESULTS}

\section{Change from Ogawa to Inaba type}

If change from Ogawa to Inaba type were to occur by mutation, as suggested by Shrivastava \& White (1947), it was essential to exclude any possibility that the antiserum used had any role other than the mechanical separation of the parent organisms from the mutants, by clumping the parents and allowing the mutants to multiply in the supernatant fluid. Before proceeding to test 
this, preliminary experiments were carried out on the same pattern as those of Shrivastava \& White (1947) to confirm this change with strains Ogawa 502 and Ogawa M 64. The results of these experiments are recorded in Tables 1 and 2.

Table 1. Ogawa $\rightarrow$ Inaba transition in Vibrio cholerae strain Ogawa 502

A culture of $V$. cholerae, strain Ogawa 502, was made in $5 \mathrm{ml}$. broth containing $1 / 50$ Ogawa $O$ antiserum (titre 1/2500). The supernatant fluid was plated out at intervals during incubation at $37^{\circ}$ to give discrete colonies.

\begin{tabular}{|c|c|c|c|c|c|c|c|c|c|c|c|c|c|c|c|c|}
\hline \multirow{4}{*}{$\begin{array}{c}\text { Inoculum } \\
\text { size } \\
\text { (no. of } \\
\text { organisms) }\end{array}$} & \multicolumn{16}{|c|}{ Time at which sample was plated } \\
\hline & \multicolumn{4}{|c|}{$2 \mathrm{hr}}$. & \multicolumn{4}{|c|}{$4 \mathrm{hr}$} & \multicolumn{4}{|c|}{$7 \mathrm{hr}}$. & \multicolumn{4}{|c|}{$24 \mathrm{hr}$. } \\
\hline & \multirow[t]{2}{*}{$\begin{array}{c}\text { No. of } \\
\text { colonies }\end{array}$} & \multicolumn{3}{|c|}{$\begin{array}{l}\text { Type of } \\
\text { colony* }\end{array}$} & \multirow[t]{2}{*}{$\begin{array}{l}\text { No. of } \\
\text { colonies }\end{array}$} & \multicolumn{3}{|c|}{$\begin{array}{c}\text { Type of } \\
\text { colony }\end{array}$} & \multirow[t]{2}{*}{$\begin{array}{c}\text { No. of } \\
\text { colonies }\end{array}$} & \multicolumn{3}{|c|}{$\begin{array}{c}\begin{array}{c}\text { Type of } \\
\text { colony }\end{array} \\
\end{array}$} & \multirow[t]{2}{*}{$\begin{array}{c}\text { No. of } \\
\text { colonies }\end{array}$} & \multicolumn{3}{|c|}{$\begin{array}{c}\begin{array}{c}\text { Type of } \\
\text { colony }\end{array} \\
\end{array}$} \\
\hline & & In & $\mathrm{Og}$ & $\mathbf{r}$ & & In & $\mathrm{Og}$ & $r$ & & In & $\mathrm{Og}$ & $\mathbf{r}$ & & In & $\mathrm{Og}$ & $\mathbf{r}$ \\
\hline $8 \times 10^{9}$ & 80 & - & 80 & - & 80 & 1 & 79 & - & 80 & - & 79 & 1 & 40 & 39 & - & $\mathbf{1}$ \\
\hline $8 \times 10^{7}$ & 80 & - & 80 & - & 80 & - & 80 & - & 80 & - & 80 & - & 36 & 22 & 8 & 6 \\
\hline $8 \times 10^{5}$ & 80 & - & 80 & - & 80 & - & 80 & - & 80 & - & 80 & - & 40 & - & 14 & 26 \\
\hline $8 \times 10^{8}$ & 80 & - & 80 & - & 80 & - & 80 & - & 80 & - & $79 \dagger$ & - & 40 & - & 4 & 36 \\
\hline
\end{tabular}

Table 2. Ogawa $\rightarrow$ Inaba transition in Vibrio cholerae strain Ogawa $M 64$

A culture of $V$. cholerae, strain M64 in $5 \mathrm{ml}$. broth containing $1 / 50$ Ogawa $O$ serum (titre 1/2500). Supernatant fluid plated out at intervals for discrete colonies (see Table 1).

Time of plating during incubation at $37^{\circ}$

\begin{tabular}{|c|c|c|c|c|c|c|c|c|c|c|c|c|c|c|c|c|}
\hline \multirow{3}{*}{$\begin{array}{l}\text { Inoculum } \\
\text { size } \\
\text { (no. } \\
\text { organisms) }\end{array}$} & \multicolumn{4}{|c|}{$2 \mathrm{hr}$} & \multicolumn{4}{|c|}{$4 \mathrm{hr}}$. & \multicolumn{4}{|c|}{$7 \mathrm{hr}}$. & \multicolumn{4}{|c|}{$24 \mathrm{hr}}$. \\
\hline & \multirow{2}{*}{$\begin{array}{l}\text { No. of } \\
\text { colonies }\end{array}$} & \multicolumn{3}{|c|}{$\begin{array}{l}\text { Type of } \\
\text { colony }\end{array}$} & \multirow{2}{*}{$\begin{array}{l}\text { No. of } \\
\text { colonies }\end{array}$} & \multicolumn{3}{|c|}{$\begin{array}{c}\begin{array}{c}\text { Type of } \\
\text { colony }\end{array} \\
\end{array}$} & \multirow{2}{*}{$\begin{array}{l}\text { No. of } \\
\text { colonies }\end{array}$} & \multicolumn{3}{|c|}{$\begin{array}{c}\text { Type of } \\
\text { colony }\end{array}$} & \multirow{2}{*}{$\begin{array}{l}\text { No. of } \\
\text { colonies }\end{array}$} & \multicolumn{3}{|c|}{$\begin{array}{l}\text { Type of } \\
\text { colony }\end{array}$} \\
\hline & & In & $\mathrm{Og}$ & $\mathrm{r}$ & & In & $\mathrm{Og}$ & $\mathbf{r}$ & & In & Og & $\mathbf{r}$ & & In & Og & $\mathbf{r}$ \\
\hline $3.5 \times 10^{9}$ & 40 & - & 40 & - & 40 & - & 40 & - & 40 & - & 40 & - & 40 & 40 & - & - \\
\hline $3.5 \times 10^{7}$ & 40 & - & 40 & - & 40 & - & 40 & - & 40 & - & 40 & - & 40 & 39 & 1 & - \\
\hline $3.5 \times 10^{5}$ & 40 & - & 40 & - & 40 & - & 40 & - & 40 & - & 40 & - & 41 & 1 & 8 & 32 \\
\hline $3.5 \times 10^{3}$ & 40 & - & 40 & - & 40 & - & 40 & - & 40 & - & 37 & 3 & 40 & - & 9 & 31 \\
\hline
\end{tabular}

These experiments studied the effect of decreasing size of inoculum and the period of incubation in Ogawa-antiserum broth required for the isolation of Inaba mutants. Twenty-four hr. cultures of the strains were suspended in saline, and 100-fold dilutions made in broth. From the undiluted suspension and from each dilution $1 \mathrm{ml}$. samples were transferred to $5 \mathrm{ml}$. broth containing $1 / 50$ Ogawa $\mathrm{O}$ antiserum (titre 2500) and the supernatant fluid plated out to give discrete colonies after different periods of incubation. The types of colonies thus obtained were tested by Stocker's sloppy-agar technique, with Ogawa $\mathrm{O}$ antiserum in the sloppy layer. Mutants which were isolated by this method were checked later by slide-agglutination tests. 'Compact' colonies were re-examined to ascertain whether they were rough or non-motile mutants. 
The supernatant cultures of both strains after incubation for $24 \mathrm{hr}$. were predominantly of the Inaba type when the inoculum was greater than $10^{7}$ organisms. When the inoculum was $10^{5}$ organisms, Inaba mutants did not appear with Ogawa 502 (Table 1) while only one of 41 colonies was of the Inaba type in Ogawa M 64 (Table 2). With an inoculum of $10^{3}$ organisms, Inaba mutants did not appear with either strain. The frequency of appearance of rough mutants was in the reverse order, being most frequent with the smaller inocula. Further, the period of incubation was important as only a few mutants could be isolated from earlier samples, but they predominated after $24 \mathrm{hr}$.

These findings, though not conclusive, are consistent with the mutation hypothesis. With larger inocula mutants were probably introduced into the culture at the start and the time-lag indicated the period required for mutants to become predominant in the supernatant culture. It was also possible that smaller inocula, which were presumably devoid of mutants at the time of implant, grew in clumps in the depths of the medium under conditions unfavourable for active multiplication and for the expression of mutants. The predominance of rough mutants with the smaller inocula appeared to indicate that this mutation was more frequent than the change Ogawa $\rightarrow$ Inaba. The apparent absence of rough forms in the presence of Inaba mutants is difficult to explain and was probably due to smooth and rough strains of Vibrio cholerae having different growth rates as observed by Bhaskaran (1953).

Proof of the presence of Inaba mutants in Ogawa cultures before serum treatment would require their detection in the parent culture. Judging from these experiments this might be possible if at least $10^{7}$ colonies of the parent culture were examined. As this was not practicable it was necessary to resort to procedures which might be expected to simplify the study.

Treatment with ultraviolet irradiation. As ultraviolet irradiation is known to increase mutation rates in bacteria, this treatment was used to see whether Inaba mutants could then be isolated from Ogawa cultures without the aid of antiserum. A volume (10 ml.) of a saline suspension of strain Ogawa 502 was

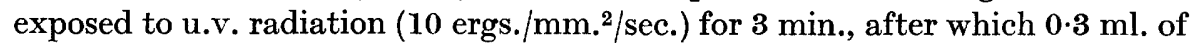
the irradiated culture was grown in broth for $4 \mathrm{hr}$. to allow the phenotypic expression of the mutants. The culture was then plated out on several agar plates to give discrete colonies and each colony thus obtained was typed by Stocker's sloppy-agar method. It was possible in this way to examine a total of 10,812 discrete colonies; of these 4 were rough, the rest being of the parent Ogawa type.

Treatment with antiserum in the cold. As the above technique failed to show Inaba mutants in the Ogawa cultures, it was necessary to revert to antiserum as a selective agent, but the experiments were now carried out in the cold to prevent the growth of the organisms, while a partial separation of the parent from mutant organisms was produced by agglutinins present in the antiserum. Pre-existing mutants would escape agglutination in the culture examined, and their numbers in relation to the colony count would indicate the approximate mutation frequencies. 
Table 3 records the results of experiments carried out in this way with three Ogawa strains of Vibrio cholerae. The test was carried out by adding $0 \cdot 1 \mathrm{ml}$. Ogawa $O$ antiserum (titre $1 / 2500$ ) to $5 \mathrm{ml}$. cold saline suspensions of each culture and keeping these in the refrigerator for $2 \mathrm{hr}$. The clumps were separated by spinning at $2500 \mathrm{rev} . / \mathrm{min}$. for $30 \mathrm{~min}$. in a refrigerated centrifuge. The supernatant fluid was then plated out to give discrete colonies. From the colony counts of the original culture and the supernatant fluid, and from the proportion of Inaba organisms detected in the latter, it was possible to estimate the approximate number of Inaba mutants in the culture. Table 3 shows that the population was decreased from $10^{9}$ organisms $/ \mathrm{ml}$. to $10^{5}$ or $10^{6}$ organisms $/ \mathrm{ml}$. as a result of agglutination with antiserum and subsequent centrifugation. The number of Inaba mutants isolated in relation to the number of colonies examined from the supernatant fluid indicated that in the original culture Inaba mutants could not be present at less than 1 in $4.8 \times 10^{6}$, 1 in $1.3 \times 10^{7}$, and 1 in $10^{7}$ organisms in the three strains examined.

Table 3. Detection of Inaba mutants in Ogawa type cultures of Vibrio cholerae

\begin{tabular}{|c|c|c|c|c|c|}
\hline \multirow{3}{*}{$\begin{array}{c}\text { Strains } \\
\text { Jgawa M64 }\end{array}$} & \multicolumn{2}{|c|}{ Colony count } & \multirow{2}{*}{$\begin{array}{l}\text { No. of } \\
\text { colonies } \\
\text { examined }\end{array}$} & \multirow[b]{2}{*}{$\begin{array}{l}\text { No. of } \\
\text { mutants }\end{array}$} & \multirow{2}{*}{$\begin{array}{c}\text { Frequency of } \\
\text { mutant in } \\
\text { parent } \\
\text { culture }\end{array}$} \\
\hline & $\begin{array}{l}\text { Original } \\
\text { culture }\end{array}$ & $\begin{array}{l}\text { Supernatant } \\
\text { fluid }\end{array}$ & & & \\
\hline & $1.6 \times 10^{9} / \mathrm{ml}$ & $1.3 \times 10^{6} / \mathrm{ml}$ & 7800 & 2 Inaba 1 Ogawa & $1: 4.8 \times 10^{6}$ \\
\hline Ogawa M $\mathbf{7 0}$ & $1.9 \times 10^{9} / \mathrm{ml}$ & $2 \cdot 4 \times 10^{5} / \mathrm{ml}$ & 5000 & 4 Inaba 1 rough & $1: 1 \times 10^{7}$ \\
\hline Ogawa M76 & $2.6 \times 10^{9} / \mathrm{ml}$ & $6.5 \times 10^{5} / \mathrm{ml}$ & 3250 & 6 rough & $1: 1.3 \times 10^{7}$ \\
\hline
\end{tabular}

There was scope for error in these estimates. It was inevitable that during centrifugation some of the mutant organisms might be deposited along with the agglutinated clumps of the parent culture. The extent to which this occurred was revealed by model experiments, carried out under identical conditions, and using a mixture of known viable populations of the two types. This showed that only $2 \%$ of the Inaba organisms in the mixture was accounted for. A source of error even in these model experiments was the almost certain presence of Inaba mutants introduced with the Ogawa culture, which could not be taken into account. These facts emphasized that estimates of the ratio mutant: parent, as recorded in Table 3, could only be approximate even when a correction was applied on the basis of the model experiments. With the correction $(50 \times)$ the approximate number of Inaba mutants in Ogawa cultures would be 1 in $10^{5}$ in strain Ogawa M 64, 1 in $2 \times 10^{5}$ in strain Ogawa $\mathrm{M} 70$, and 1 in $2 \times 10^{5}$ in Ogawa $\mathrm{M} 76$.

\section{Possibility of Inaba to Ogawa mutation}

Shrivastava \& White (1947) observed that a change from Inaba to Ogawa could not be demonstrated in Inaba strains under conditions similar to those which facilitated the isolation of Inaba mutants from Ogawa cultures, that is, 
by growing them in broth containing type-specific antiserum. This question was re-examined, as it was possible that with Stocker's sloppy-agar technique the change might be demonstrable. Experiments were carried out with four Inaba strains: Inaba 8024, Inaba 53/54 and Inaba 55/54 (wild strains) and strain B 40 an Inaba mutant of Ogawa M 70 isolated in a previous experiment. When these strains were grown in broth containing Inaba $\mathbf{O}$ antiserum for $\mathbf{2 4} \mathrm{hr}$., and the supernatant fluid plated out to give discrete colonies, numerous rough colonies were seen but none was of the Ogawa type. With the intention to decrease the predominance of rough variants, in one experiment 'rough' antiserum was also added to the broth. This decreased the number of rough colonies obtained from the supernatant fluid but did not reveal Ogawa-type colonies. Serial cultivations in these antiserum media did not result in Ogawa mutants either.

\section{Rough and non-motile mutants of Vibrio cholerac}

The Stocker sloppy-agar technique, while applicable to type determination of Vibrio cholerae, could also be used for the identification of rough and nonmotile mutants. With antiserum-free sloppy agar 4 smooth motile strains were examined for the frequency of appearance of rough or non-motile colonies. Table 4 shows that rough and non-motile mutants appeared with a greater frequency than the antigenic-type mutations studied earlier, particularly in strain Inaba 243 ( 1 rough mutant in every 1300 colonies and 1 non-motile mutant in every 2000 colonies). The frequency of these mutations in the other strains varied from 1 in $1 \cdot 4 \times 10^{4}$ to 1 in $1 \cdot 2 \times 10^{4}$ for non-motile mutants, and from 1 in $9 \times 10^{3}$ to 1 in $4 \times 10^{3}$ for rough mutants. From these findings it seems safe to conclude that these mutations were of the order of 1 in $10^{4}$ divisions or less.

Table 4. Rough and non-motile mutants of Vibrio cholerae

$\begin{array}{lccc}\text { Strain } & \begin{array}{c}\text { No. of } \\ \text { colonies } \\ \text { examined }\end{array} & \begin{array}{c}\text { No. of } \\ \text { rough } \\ \text { mutants }\end{array} & \begin{array}{c}\text { No. of } \\ \text { non-motile } \\ \text { mutants }\end{array} \\ \text { Inaba 8024 } & 9,100 & 1 & 0 \\ \text { Inaba 243 } & 12,000 & 9 & 6 \\ \text { Ogawa 502 } & 14,200 & 2 & 1 \\ \text { Ogawa 8022 } & 12,000 & 3 & 1\end{array}$

\section{DISCUSSION}

Antigenic variants of Vibrio cholerae have been demonstrated in the presence of immune serum or phage (Yang \& White, 1934; Shrivastava \& White, 1947). It is often inferred that these agents induced the observed changes in the organism. References in the literature to 'converted' Inaba strains (meaning Inaba strains isolated from Ogawa cultures) are instances of this belief. Shrivastava \& White (1947) were, however, careful to point out that any attack on the surface of a cell could not be expected to induce transmissible modifications. They also indicated that these changes might be due to loss variations during growth. The basic difficulty in determining whether a change 
is due to mutation or not, is the difficulty of examining a large number of bacteria for the existence of organisms with the variant character without using selective methods which might act directly on the organisms. If this difficulty could be overcome, their mutational origin could be established. It was with this in view that the sloppy-agar technique described by Stocker (1949) was adapted for the present study. Type differentiation of a large number of colonies was possible by this means, and in all three cases the time of exposure to the antiserum in the sloppy agar was too short to induce variants. These studies established that the rough and non-motile variants were always present in the parent culture and hence were due to mutations, which appeared more or less with the same frequency in each case (about 1 in 10,000 colonies examined). It may be mentioned that Yang \& White (1934) were successful only once in demonstrating the existence of rough mutants in smooth cultures by the ingenious procedure of washing plated cultures of smooth strains with $2 \% \mathrm{NaCl}$ solution and examining the adherent colonies which survived the treatment. As regards non-motile mutants, their direct demonstration in populations of motile strains of Vibrio cholerae has not hitherto been reported. However, the existence of such strains was known; Balteanu (1926) found that they were associated with opaque colony character.

Unequivocal evidence for antigenic type variation in Vibrio cholerae as a result of mutation could not be obtained. However, experiments with antisera carried out in the cold confirmed that growth of the culture subsequent to the addition of specific antiserum was not necessary for the isolation of Inaba mutants from Ogawa cultures. This makes it very probable that these mutants were already present in the parent culture, and agglutination by antiserum only facilitated isolation of the mutant in the supernatant fluid. The rate of appearance of these mutants was of the order of 1 in $10^{5}$ cell-divisions. The inability to demonstrate directly the presence of these mutants in Ogawa cultures was probably due to this low mutation rate, which would require the examination of a similarly large number of colonies for a chance isolation of the mutant. This was obviously not possible even with the sloppy-agar technique.

The failure to isolate Ogawa mutants from Inaba cultures with the aid of selective antiserum, may have been the result of still lower reverse mutation rates. Further, only single-step mutations can be revealed by the tests used and perhaps the change Inaba $\rightarrow$ Ogawa type involves multi-step mutation. The occurrence of Hikojima type, intermediate between Inaba and Ogawa, as well as the identification of imperfect Inaba strains (containing demonstrable Ogawa antigenic factor) by Shrivastava $\&$ White (1947), may be of significance in this connexion. It would be interesting to inquire into the origin of these Hikojima strains. Either they occurred as a result of a partial carry-over of Ogawa antigen in Inaba mutants, or they were due to a reverse change from Inaba to Ogawa type by successive mutations, accompanied by a progressive replacement of the $\mathrm{C}$ antigen of Inaba by the $\mathrm{B}$ antigen of Ogawa. Proof of this might be possible if such intermediate types could be isolated from pure Inaba or Ogawa strains; in the absence of any selective method this may be 
difficult. This multi-step mutational hypothesis may also explain the occurrence of strains of trivalent agglutinability with Inaba, Ogawa and 'rough' antisera (Shrivastava \& White, 1947; Bhaskaran, 1953).

The loss-variation hypothesis of Shrivastava \& White (1947) is an alternative explanation for the non-reversion of Ogawa to Inaba mutation. They suggested that the transition from Ogawa to Inaba type may be due to failure of the organism to synthesize certain chemical groupings, so that the reverse change would be one of synthetic revival which might be difficult to achieve. No differences were, however, seen in the nutritional requirements of Ogawa and Inaba type strains of Vibrio cholerae (Bhaskaran \& Rowley, 1956), though the differences may not be so gross as to be revealed by the auxanographic methods used in that study, which showed a general requirement for purines. More detailed knowledge of the synthesis of purines by $\boldsymbol{V}$. cholerae and the role of certain amino acids which are essential nutrients for some Inaba strains, may show whether there is any difference in this aspect of the synthetic processes in the two types.

We wish to thank Professor R. Cruickshank for his interest and support. We are also grateful to Dr B. A. D. Stocker and Dr D. Rowley for their helpful suggestions. Strains of Vibrio cholerae were kindly made available to us by the Directors of various laboratories in India and the National Collection of Type Cultures, Colindale Avenue, London, N.W. 9. This work was carried out when one of us (K.B.) was holding a Fellowship under the Nuffield Foundation in the United Kingdom.

\section{REFERENCES}

Balteanu, I. (1926). The receptor structure of Vibrio cholerae with observations on variations in cholera and cholera-like organisms. J. Path. Bact. 29, 251.

Bhaskaran, K. (1953). Studies on vibrio dissociation. Part I. Smooth-rough dissociation of $V$. cholerae in rosaniline agar. Indian $J$. med. Res. 41, 143.

Bhaskaran, K. \& Rowley, D. (1956). Nutritional studies on Vibrio cholerae. J. gen. Microbiol. 15, 417 .

Gardner, A. D. \& Venkatraman, K. V. (1935). The antigens of the cholera group of vibrios. J. Hyg., Camb. 35, 262.

Gorrill, R. H. \& GRAY, R. A. (1956). The induction of bacteriophage in staphylococci. J. gen. Microbiol. 14, 167.

Kabeshima, T. (1918). Immunological properties of the cholera bacillus. C.R. Soc. Biol., Paris, 81, 618.

Kauffmann, F. (1950). On the serology of Vibrio cholerae. Acta path. microbiol. scand. 27, 282.

Nobechi, K. (1923). Immunological studies upon types of Vibrio cholerae. Sci. Rep. Inst. infect. Dis. Tokyo Univ. 2, 43.

Shrivastava, D. L. \& White, P. B. (1947). Note on the relationship of the socalled Ogawa and Inaba types of $V$. cholerae. Indian med. Res. 35, 117.

Stocker, B. A. D. (1949). Measurements of the rate of mutation of flagellar antigenic phases in Salmonella typhimurium. J. Hyg., Camb. 47, 398.

Venkatraman, K. V. (1947). A note of the re-appearance of the Inaba type of $V$. cholerae in the Madras Province. Report of the Scientific Advisory Board for the year 1947, Indian Research Fund Association, New Delhi.

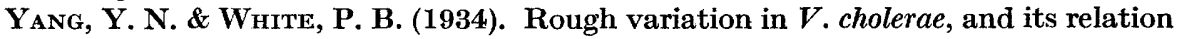
to resistance to choleraphage (type A). J. Path. Bact. 38, 187.

(Received 28 December 1956) 